\title{
Awareness of Myopia: A Survey Among Parents Residing in Singapore
}

Arief Tjitra Salim

Plano Pte Ltd https://orcid.org/0000-0001-9256-6818

Stuart Keel

Centre for Eye Research Australia

Joshua Foreman

Plano Pte Ltd

Mohamed Dirani ( $\triangle$ mo.dirani@plano.co )

Research article

Keywords: Myopia; Survey; Awareness

Posted Date: June 17th, 2019

DOl: https://doi.org/10.21203/rs.2.10314/v1

License: (c) (i) This work is licensed under a Creative Commons Attribution 4.0 International License. Read Full License 


\section{Abstract}

Background Given the rising public health burden of myopia, population-based data on the level of awareness about myopia amongst parents in Singapore are required for planning future awareness campaigns and public health interventions. This study aimed to evaluate the level of awareness of myopia amongst a group of parents residing in Singapore. Methods This study was a cross-sectional survey. A sample of 326 participants aged 19 to 69 years with at least 1 child were recruited at The Kidz Academy (TKA) 2018 exhibition in Singapore. Socio-demographic characteristics, smart device use and awareness of myopia (prevalence and mitigation strategies) were collected via an intervieweradministered online questionnaire. Results $56.7 \%$ (185/326) of survey respondents were aware of the high prevalence of myopia amongst secondary school-going Singaporean children. 18.1\% (59/326) of the respondents reported that an eye-to-screen distance of less than $30 \mathrm{~cm}$ was acceptable and $27.0 \%$ $(88 / 326)$ were unaware of the importance of regular eye examinations among children. Less than half of the respondents $[46.6 \%(152 / 326)]$ correctly identified that Singaporean children spend at least 6.5 hours a day on digital screens. $87.7 \%(286 / 326)$ were aware of the importance of outdoor activity as an intervention to slow the progression of myopia, with female respondents significantly more likely to be aware of its importance [odds ratio $(O R)=2.12,95 \%$ confidence interval $=1.09-4.13, \chi 2=4.86, P=.03$ ] Conclusion Survey respondents demonstrated a good level of awareness of the high prevalence of myopia in Singapore and the protective role of outdoor activity in its development and progression. Awareness campaigns may be beneficial for promoting regular eye examinations amongst children and educating the public on the risk of excessive time spent on smart devices.

\section{Background}

Myopia is a major global public health and economic problem. An estimated 1.95 billion people worldwide have myopia and this figure is expected to increase to 5 billion by 2050 [1]. In Singapore alone, estimates suggest that the prevalence of myopia is as high as $11 \%$ amongst children aged $6-72$ months [2] and over 70\% in those undertaking college education [3]. Moreover, myopia poses a considerable financial burden, with estimated annual direct costs in Singapore at US\$755 million, and globally at USD\$328 billion [4].

The causes of myopia are both genetic and environmental $[5,6]$, with leading modifiable environmental risk factors including increased near work activity and reduced time spent outdoors during childhood [79]. As a case in point, a recent randomised controlled trial reported that children who were engaged in one 40-minute class outdoors per day and whose parents encouraged more outdoor activity after school had a 9.1\% lower incidence of myopia compared to normal controls [9]. In Singapore, strategies to modify environmental risk factors of myopia through improved education and awareness as well as school screening programs have been successful in reducing the prevalence of myopia by $5 \%$ in primary school children $[10,11]$. A study in China also reported that children had a lower risk of developing myopia if their parents paid attention to their vision from a young age [12], highlighting the indispensable role that parents play in myopia management. 
There is currently a paucity of data investigating the awareness of myopia and its management strategies amongst parents worldwide. One study in Ireland evaluated the awareness and attitudes of 329 parents with children aged $8-13$ years and reported that only $46 \%$ considered myopia to be a health concern and only $14 \%$ showed concern about their children developing myopia [13]. To our knowledge, no study has assessed the awareness of myopia amongst parents residing in Singapore. Given the rising prevalence of myopia in Singapore and the critical role parents will play in addressing this worsening epidemic, understanding their level of awareness of myopia risks, prevention and management will prove indispensable to public health programmes for myopia. Herein, we present the observations of a survey of parental awareness of myopia, its management strategies, and smart device usage amongst parents residing in Singapore.

\section{Methods}

\section{Participant recruitment}

Parents with at least one child aged 16 years or younger who attended the Kidz Academy (TKA) exhibition in Singapore (29 June to 1 July 2018) were invited to complete the questionnaire. Eligible participants provided written informed consent before administration of the questionnaire. Principles of the Declaration of Helsinki were observed.

\section{Questionnaire}

An interviewer-administered online questionnaire (Google form) was developed by the research team. The questionnaire consisted of 13 items that were separated into two domains. The first domain captured the participants' socio-demographic information ( 5 items) and evaluated basic awareness of myopia and eye care management strategies (4 items). The second domain recorded their children's demographics and past ocular history (4 items).

\section{Socio-demographic items}

Participant socio-demographic information included age, gender, race, highest educational qualification and number of children ( 5 items). The questionnaire also recorded the age of the participants' children and the children's last eye examination period. The first 2 items enquired about the age and last eye examination period of the first child, while the remaining 2 items enquired about the other child/children's age and last eye examination period.

\section{Myopia knowledge and awareness items}

The questionnaire contained 4 items on the participants' myopia awareness, consisting of 3 four-option multiple choice items with 1 correct answer and 1 four-option item with more than 1 correct answer: 
The participants were asked to state what they believed the prevalence of myopia was in secondary school-going Singaporean children by selecting one of the following percentages: $30 \%, 50 \%, 75 \%$ or $90 \%$. The correct answer of 75\% was based on data from the Singapore Health Promotion Board (HPB) in 2011 [11].

The next item required the participants to state what they believed was the average amount of time 12year old children in Singapore spend on devices daily by selecting one of the following options: 2 hours, 4.5 hours, 6.5 hours or 8 hours. The correct answer was 6.5 hours based on a report from the DQ Institute [14].

The participants were then asked on their awareness of the minimum safe distance of device screens from the eyes, by requiring them to select one of the following options: $10 \mathrm{~cm}, 20 \mathrm{~cm}, 30 \mathrm{~cm}$ or $40 \mathrm{~cm}$. The correct answer was $30 \mathrm{~cm}$ based on studies investigating the average working distance when using phones, and that closer working distances were associated with adverse ocular symptoms $[15,16]$.

The last item which had multiple correct answers was related to participant awareness of strategies to prevent the onset and slow the progression of myopia, with the correct answer being all four of the proposed strategies, including outdoor activity and natural light exposure, looking far into the distance, taking regular breaks and undergoing annual eye check-ups.

\section{Statistical analysis}

De-identified data were downloaded from the Google form server and imported into SPSS statistics software version 25.0 (IBM Corporation, United States). A $P$-value of .05 was used for significance testing. Descriptive statistics were used to summarise the demographic characteristics of the survey population and logistic regression tests were utilised to compare the questionnaire responses. Multinomial logistic regression was used to assess the relationship between the demographic variables and participants' awareness of the prevalence of myopia, recommended eye-to-screen distance and average daily time spent on devices. Binomial logistic regression was utilised to investigate the association between the demographic variables and knowledge of the effective strategies to prevent the onset and slow the progression of myopia. Odds ratios (OR) and 95\% confidence intervals $(\mathrm{Cl})$ were also calculated for risk factors that were significantly and independently associated with the participants' myopia awareness.

\section{Results}

\section{Parent demographics}

Of the 605 individuals who were invited to complete the questionnaire, a total of 326 (53.9\%) agreed to participate (Table 1). The sample of the participants who completed the questionnaire had a mean age of 37.17 .9 years and $63.5 \%(207 / 326)$ were female. The majority of the participants were of Chinese 
ethnicity $(71.8 \%, 234 / 326)$, and $46.3 \%(151 / 326)$ had completed a Bachelor's degree. The mean number of children per family was 1.70 .8 , with nearly half $(46.6 \%, 152 / 326)$ having only 1 child.

\section{Myopia awareness and knowledge}

Fifty-seven percent of participants (185/326) correctly indicated the prevalence of myopia in secondary school-going children in Singapore to be $75 \%$ (Table 2). Over half of all participants $(53.4 \%, 174 / 326)$ underestimated the time spent on devices amongst primary school-aged children in Singapore (i.e. 6.5 hours daily). Fifty-nine participants (18.1\%) indicated that an eye-to-screen distance of less than $30 \mathrm{~cm}$ was acceptable, while 14 participants (4.3\%) reported that $10 \mathrm{~cm}$ was acceptable. Almost 9 in 10 (87.7\%, 286/326) participants identified outdoor activity and natural light exposure as effective strategies in preventing the onset and progression of myopia. In addition, $75.2 \%(245 / 326)$ and $82.5 \%(269 / 326)$ of the participants, respectively, indicated that looking into the distance and taking regular breaks from device use were important strategies in managing myopia onset and progression. Nearly three-quarters $(73.0 \%, 238 / 326)$ of participants reported that regular eye check-ups were important for children. Overall, 204 participants $(62.6 \%)$ indicated that all four management strategies were effective in mitigating myopia.

After adjusting for covariates (age, gender and highest educational attainment), logistic regression analysis revealed no significant associations between participant demographics and the awareness of the prevalence of childhood myopia in Singapore, awareness of the average daily time spent on devices amongst Singaporean children, or the appropriate eye-to-screen distance (Table 3). Females were more likely to be aware of the effectiveness of outdoor activity in delaying the onset and reducing the progression of myopia $(\chi 2=4.86, P=.03)$.

\section{Children demographics and eye check-ups}

Among the 326 participants, we captured demographic data of 524 children with a mean age of 6.13 .9 years (Table 4). Data on the utilisation of eye health care services was complete for $462(88.2 \%)$ children. Of these, participants self-reported that nearly half $(48.7 \%, 225 / 462)$ of the children had undertaken an eye examination within the last 12 months, while 180 (39.0\%) children had never had a previous eye examination.

\section{Discussion}

This survey evaluated the level of awareness of childhood myopia and its management strategies amongst a group of Singapore-based parents. A decent overall level of awareness was observed amongst the participants, with almost $60 \%$ being aware of the high prevalence of myopia amongst Singaporean children, and a similar proportion (87.7\%) being aware that outdoor activity and natural light 
exposure were effective management strategies for myopia. Nevertheless, a significant proportion (39.0\%, 180/462) of the sampled child population had never had an eye examination.

Previous surveys conducted in China [12] and Ireland [13] have highlighted the importance of parental awareness of myopia risk factors in the control of the condition. Of particular interest, Zhou and coworkers (2017) reported that positive parental attitudes and behaviours towards their children's vision, such as ensuring sufficient sleep and monitoring device usage, were associated with a delayed onset and reduced progression of myopia among Chinese children [12]. Despite this, overall parental awareness of some key risk factors, such as excessive near work activity and increased screen time, were found to be lacking in these previous reports. This is in line with findings from the present survey that over $50 \%$ of the participants were unaware that the average child's screen time was 6.5 hours per day, and $18.1 \%$ of participants considered an eye-to-screen distance of less than $30 \mathrm{~cm}$ to be acceptable.

In Singapore, the National Myopia Prevention Programme (NMPP) promotes myopia screening in preschool to secondary school students, assisting in the early detection of myopia and raising parental awareness. The impact of the NMPP has recently been highlighted by the HPB of Singapore, with a study in 2011 reporting a 5\% reduction in the prevalence of myopia among Singaporean primary school children between 2005-2011 [11]. Our finding of a good overall level of myopia awareness amongst the participants may also be a reflection of the positive impact of this programme. Nonetheless, the finding in the present survey that nearly $40 \%$ of participating Singaporean children had never undertaken an eye examination is noteworthy. Given that guidelines from the Singapore Health Services (SingHealth) recommend that children aged 16 years and under should have an eye examination every 1 to 2 years [17], this finding provides evidence that improved efforts may be required to address key barriers (real and perceived) to accessing regular eye examinations.

The limitations in the current survey include the relatively small and unevenly distributed sample size with respect to age, gender and educational qualification. Non-response bias may also be present, given that $46.1 \%(279 / 605)$ decided not to participate and/or did not complete the questionnaire. Sufficient information from all the non-responders was not available to quantify bias. In addition, the setting of participant recruitment could also be a potential source of bias. That is, it could be speculated that parents attending an education exhibition may be more engaged in their children's health and education, thus more likely to be aware of myopia and its risk factors. Lastly, survey responses were obtained via self-report and therefore the influence of recall bias cannot be discounted.

\section{Conclusion}

This survey provides insights into knowledge and awareness of myopia amongst a group of Singaporebased parents. The results suggest a good degree of myopia awareness and smart device use management, however further improvements in eye care utilisation amongst Singaporean children may be warranted. Given that parents play a prominent role in myopia management, additional research 
exploring awareness of myopia-related risk factors amongst a larger population of Singaporean parents may assist in guiding more targeted public awareness campaigns.

\section{Declarations}

Ethics approval and consent to participate

Eligible participants provided written informed consent before administration of the questionnaire. Principles of the Declaration of Helsinki were observed.

Consent for publication

Not applicable for this manuscript.

Availability of data and materials

The datasets used and/or analysed during the current study are available from the corresponding author on reasonable request.

Competing interests

Senior author Associate Professor Mohamed Dirani is a shareholder of the company Plano Pte Ltd, first author Arief Tjitra Salim is an employee of Plano Pte Ltd and second author Dr Stuart Keel and third author $\mathrm{Dr}$ Joshua Foreman are consultants for Plano Pte Ltd who receive reimbursements in the form of honorariums.

Funding

There are no requests for funding for this study. However, it is to be noted that Plano Pte Ltd covered the costs related to the space during the Kidz Academy (TKA) exhibition.

Authors' contributions

Senior author Associate Professor Mohamed Dirani oversaw the research study. First author Arief Tjitra Salim analysed and interpreted the survey data and was a major contributor in writing the manuscript. Second author Dr Stuart Keel and third author Dr Joshua Foreman were major contributors in reviewing the manuscript. All authors read and approved the final manuscript.

Acknowledgements

We would like to acknowledge the trained interviewers, participants and the plano team for their support at the event.

\section{References}


1. Holden, B.A., et al., Global Prevalence of Myopia and High Myopia and Temporal Trends from 2000 through 2050. Ophthalmology, 2016. 123(5): p. 1036-42.

2. Dirani, M., et al., Prevalence of refractive error in Singaporean Chinese children: the strabismus, amblyopia, and refractive error in young Singaporean Children (STARS) study. Invest Ophthalmol Vis Sci, 2010. 51(3): p. 1348-55.

3. Seet, B., et al., Myopia in Singapore: taking a public health approach. British Journal of Ophthalmology, 2001. 85(5): p. 521.

4. Organization, W.H. and B.H.V. Institute, The Impact of Myopia and High Myopia. 2015: Australia.

5. Morgan, I.G., K. Ohno-Matsui, and S.M. Saw, Myopia. Lancet, 2012. 379(9827): p. 1739-48.

6. Dirani, M., J.G. Crowston, and T.Y. Wong, From reading books to increased smart device screen time. British Journal of Ophthalmology, 2019. 103(1): p. 1.

7. Huang, H.M., D.S. Chang, and P.C. Wu, The Association between Near Work Activities and Myopia in Children-A Systematic Review and Meta-Analysis. PLoS One, 2015. 10(10): p. e0140419.

8. Dirani, M., et al., Outdoor activity and myopia in Singapore teenage children. Br J Ophthalmol, 2009. 93(8): p. 997-1000.

9. He, M., et al., Effect of Time Spent Outdoors at School on the Development of Myopia Among Children in China: A Randomized Clinical Trial. Jama, 2015. 314(11): p. 1142-8.

10. Board, H.P., Health Promotion Board Annual Report 2009/2010. 2010: Singapore.

11. Board, H.P., SINGAPORE ACHIEVES BREAKTHROUGH IN REDUCING PREVALENCE OF MYOPIA BY ALMOST 5\% 2011.

12. Zhou, S., et al., Association between parents' attitudes and behaviors toward children's visual care and myopia risk in school-aged children. Medicine (Baltimore), 2017. 96(52): p. e9270.

13. McCrann, S., et al., Parental attitudes to myopia: a key agent of change for myopia control? Ophthalmic Physiol Opt, 2018. 38(3): p. 298-308.

14. Institute, D., Cyber Risk \& Youth Empowerment in the Digital Era. 2016.

15. N., B., I Live in the Future \& Here's How It Works. 2010, New York, NY: Crown Business.

16. Bababekova, Y., et al., Font size and viewing distance of handheld smart phones. Optom Vis Sci, 2011. 88(7): p. 795-7.

17. SingHealth Regular Eye Examination. 2018. 


\section{Tables}

Table 1. Sociodemographic characteristics of participants $(n=326)$

\begin{tabular}{lll} 
Variables & & $\mathbf{n}(\%)$ \\
\hline Total number & & $326(100)$ \\
\hline Age & & \\
& Less than 29 years old & $44(13.5)$ \\
& 30 to 39 years old & $180(55.2)$ \\
\hline Gender & More than 40 years old & $102(31.3)$ \\
\hline & Male & $119(36.5)$ \\
\hline Ethnicity & Female & $207(63.5)$ \\
& & \\
& Chinese & $234(71.8)$ \\
& Malay & $9(2.8)$ \\
& Indian & $61(18.7)$ \\
\hline & Eurasian & $6(1.8)$ \\
\hline & Others & $16(4.9)$
\end{tabular}

\section{Education}

\begin{tabular}{lll}
\hline & $\begin{array}{ll}\text { Diploma or the equivalent } \\
\text { Bachelor's degree }\end{array}$ & $107(32.8)$ \\
\hline & Master's degree or higher & $68(20.9)$ \\
\hline Number of children & & \\
\hline & 1 & $152(46.6)$ \\
\hline 2 & $134(41.1)$ \\
\hline 3 & $29(8.9)$ \\
\hline 4 and above & $11(3.4)$
\end{tabular}

Table 2. Participant awareness of myopia 
In Singapore, by the time children reach secondary school, what is the prevalence of myopia?

\begin{tabular}{ll}
$30 \%$ & 24 \\
& $(7.4)$ \\
\hline $50 \%$ & 100 \\
& $(30.7)$ \\
& 185 \\
$75 \%$ & $(56.7)$ \\
& 17 \\
\hline $90 \%$ & $(5.2)$ \\
\hline
\end{tabular}

On average, how much time do 12-year children spend on devices daily?

\begin{tabular}{|c|c|}
\hline 2 hours & $\begin{array}{l}56 \\
(17.2)\end{array}$ \\
\hline 4.5 hours & $\begin{array}{l}118 \\
(36.2)\end{array}$ \\
\hline 6.5 hours & $\begin{array}{l}139 \\
(42.6)\end{array}$ \\
\hline 8 hours & $\begin{array}{l}13 \\
(4.0)\end{array}$ \\
\hline
\end{tabular}

What is considered a minimum 'safe distance' from the eyes to the screen of mobile device?

$\begin{array}{ll}10 \mathrm{~cm} & 14 \\ & (4.3) \\ 20 \mathrm{~cm} & 45 \\ & (13.8) \\ 30 \mathrm{~cm} & 220 \\ & (67.5) \\ 40 \mathrm{~cm} & 47 \\ & (14.4)\end{array}$

What are effective ways of preventing the onset or progression of myopia? (you may select more than one)

Outdoor activity and natural light exposure

Looking far into the distance 
Table 3. Logistic regression analysis for factors associated with awareness of myopia among Singaporean parents

\begin{tabular}{llllllll} 
& Age & & Gender & \multicolumn{2}{c}{ Education } \\
& Item & $\chi 2$ & $\boldsymbol{P}$ & $\chi 2$ & $\boldsymbol{P}$ & $\chi 2$ & $\boldsymbol{P}$ \\
\hline Prevalence of childhood myopia & 5.48 & .48 & 3.77 & .29 & 5.29 & .51 \\
\hline Daily time spent by children on devices & 6.30 & .39 & 2.11 & .55 & 4.94 & .55 \\
\hline Eye to screen distance & 8.50 & .20 & 1.26 & .74 & 2.80 & .83 \\
\hline Effective myopia mitigation strategies & & & & & & \\
\hline Outdoor activity and natural light exposure & 0.66 & .72 & 4.86 & .03 & 4.31 & .12 \\
\hline Looking far into the distance & 4.58 & .10 & 3.14 & .08 & 0.38 & .83 \\
\hline Taking regular breaks & 0.21 & .90 & 1.36 & .24 & 5.23 & .07 \\
\hline Annual eye check-ups & 1.26 & .53 & 0.05 & .82 & 1.95 & .38
\end{tabular}

Table 4. Children demographic and eye check-up frequency 


\begin{tabular}{lll} 
Variables & $\mathbf{n}(\%)$ & $524(100)$ \\
\hline Total number & & \\
\hline Age of children & & \\
\hline & 0 to 4 years old & $(39.7)$ \\
\hline & 5 to 9 years old & $210(40.1)$ \\
\hline & 10 to 14 years old & $95(18.1)$ \\
\hline & More than 14 years old & $11(2.1)$ \\
\hline Total number & $462(100)$ & \\
\hline Period of last eye check-up & & $180(39.0)$ \\
\hline & Never & $225(48.7)$ \\
\hline & Within the last year & $43(9.3)$ \\
\hline & Between 1 to 2 years ago & $42(2.6)$ \\
\hline & Between 2 to 3 years ago & $12(0.4)$
\end{tabular}

\section{Supplementary Files}

This is a list of supplementary files associated with this preprint. Click to download.

- supplement1.pdf 